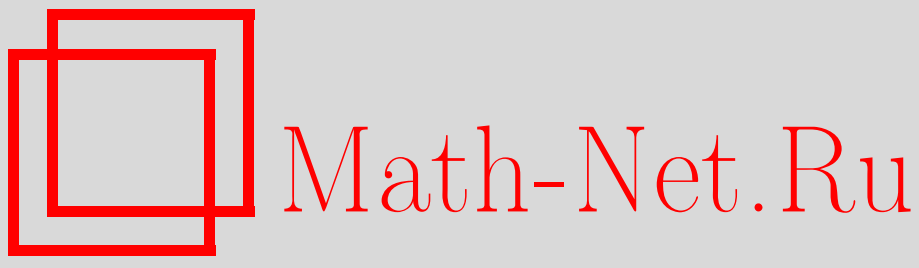

Л. В. Кудюров, Ю. К. Мустафаев, Моделирование угона рельсового пути под действием динамических нагрузок, Вестн. Сам. гос. техн. ун-та. Сер. Физ.-мат. науки, 2005, выпуск 34, 171-177

DOI: https://doi.org/10.14498/vsgtu352

Использование Общероссийского математического портала Math-Net.Ru подразумевает, что вы прочитали и согласны с пользовательским соглашением

http://www . mathnet.ru/rus/agreement

Параметры загрузки:

IP : 3.81 .55 .215

26 апреля 2023 г., 17:08:00

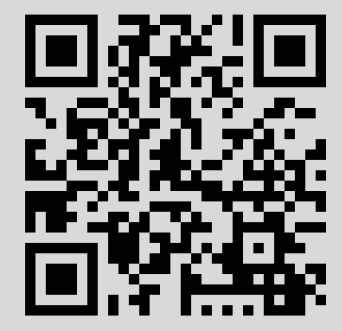




\section{МОДЕЛИРОВАНИЕ УГОНА РЕЛЬСОВОГО ПУТИ ПОД ДЕЙСТВИЕМ ДИНАМИЧЕСКИХ НАГРУЗОК}

Рассматривается динамическая модель угона железнодорожного пути в процессе его эксплуатаичи. Рельсовая нить представлена как механическая система из п элементарных участков, связанных упругими и диссипативными связями друг с другом и с основанием, на котором располагается рельсовая плеть. Применение приниипа Даламбера к выделенному элементу рельсового пути дало возможность свести задачу к системе алгебраических уравнений, позволяющих оценить угон пути в зависимости от нагрузки.

Явление угона пути, т. е. продольного перемещения рельсовой плети известно давно. Причинами угона является косой удар, испытываемый рельсом при переходе колеса через стык, температурные деформации, прогибы рельсов под действием динамических сил [1]. Последнее обстоятельство вынуждает колесо все время идти на подъем, заставляя изогнутый рельс, выпрямляясь под действием веса колеса, «выстилаться» впрямую, вызывая тем самым перемещение рельса и упругие деформации рельсовых креплений и подрельсового основания в направлении движения (рис. 1).

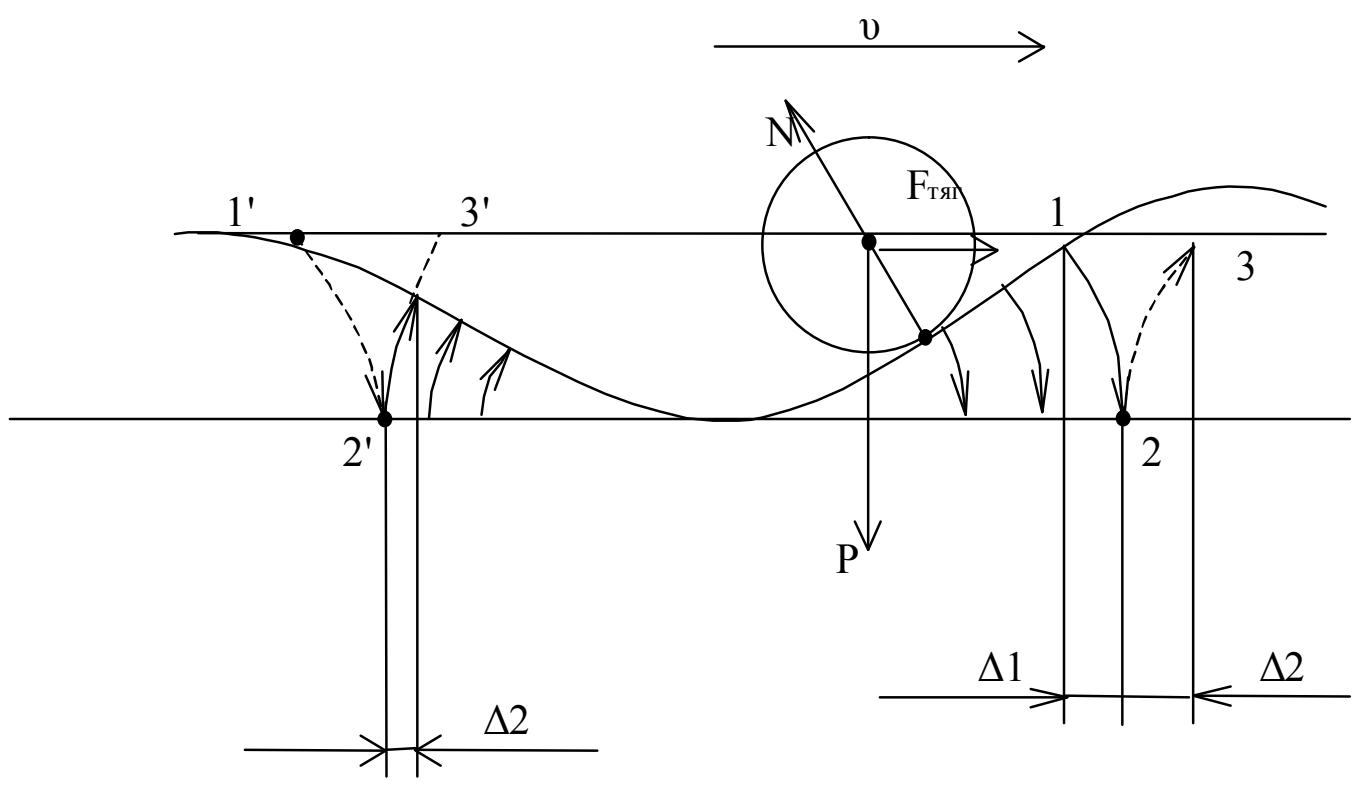

Р и с. 1. Процесс «выстилания» рельса перед набегающим колесом

Из всех указанных причин особый интерес представляет последняя, так как она обусловлена только динамическими факторами. Особую актуальность эта гипотеза приобретает при рассмотрении бесстыкового пути.

Существующие методы расчета динамического угона пути достаточно сложны и требуют решения систем дифференциальных уравнений в частных производных. При этом возникают сложности вычислительного характера, связанные с необходимостью учета большого числа движущихся сил, равных количеству колесных пар состава. Поэтому представляет интерес разработка эффективного алгоритма вычислений, отличающегося быстродействием и достоверностью расчетов.

Для реализации поставленной задачи была построена модель, использующая следующие допущения:

рельс рассматривается как система элементов, связанных друг с другом и с основанием упругими связями (рис. 2);

каждый элемент представляет собой материальную точку, объединяющую в себе шпалу и участок рельса над ней, длина которого равна длине междушпального расстояния; масса эле- 
мента равна суммарной массе шпалы, рельсовых скреплений и заменяемого участка рельса (рис. 3);

возмущения не передаются мгновенно от элемента к элементу; существует определённая скорость распространения возмущения (для металлов - скорость звука, равная примерно $5000 \mathrm{M} / \mathrm{c})$.

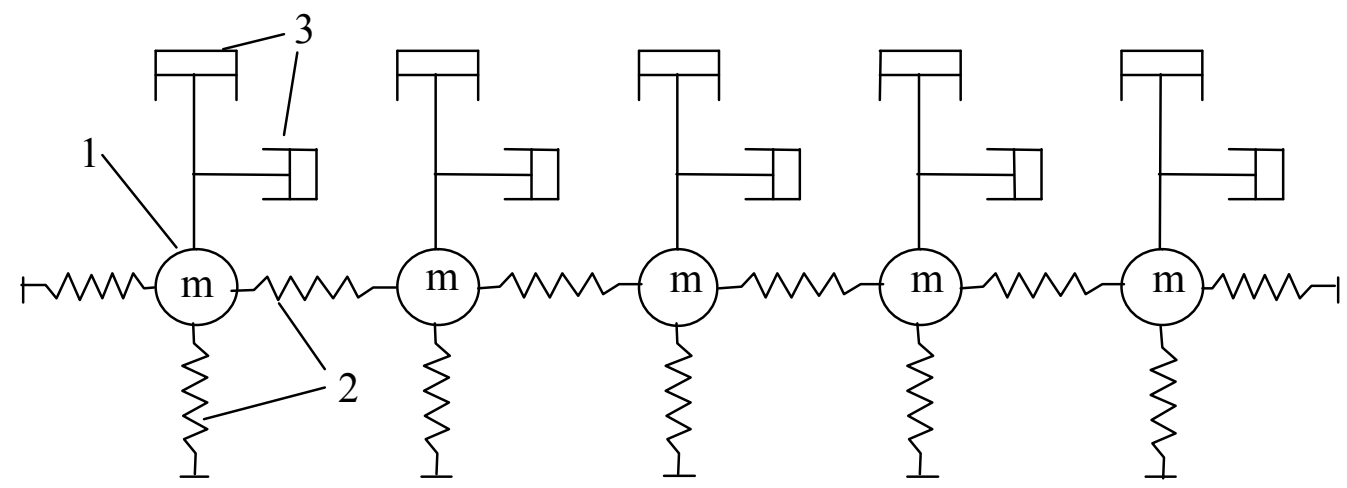

Р и с.2 Модель рельсовой плети, состоящая из отдельных элементов:

1 - элементы массой $m$, равной массе моделируемого участка; 2 - упругие связи; 3 - демпферы

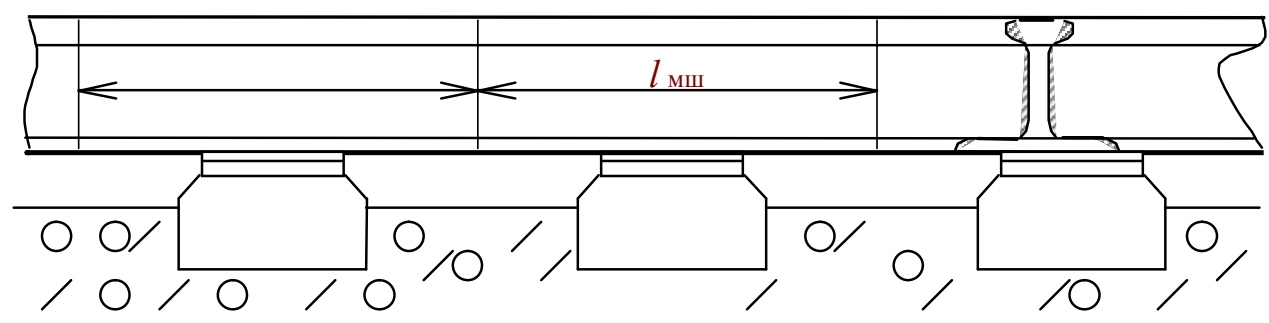

Р и с. 3. Разбиение рельсового пути на элементарные участки

Очевидно, что данная система склонна к распространению колебательных (волновых) процессов. Наличие конечной скорости распространения возмущающего воздействия позволяет рассматривать выделенный участок рельса на коротком промежутке времени как отдельную изолированную колебательную систему. Интервалы времени должны быть таковыми, чтобы изменение характеристик системы происходило с достаточной плавностью (не более 3 - 5 \% от любого параметра). Исходя из заданной точности, нетрудно подсчитать, что при чистом синусоидальном колебательном процессе необходимо делать 200 выборок на период (примерно 3\% точность).

Учитывая, что длина междушпального промежутка равна примерно 0,5 м, а скорость распространения волны 5000 м/с, а также то, что нет физического смысла в волне, длина которой меньше междушпального расстояния (нет опоры для колебаний), делаем вывод, что необходимые характеристики должны быть следующими:

интервал времени (период волны) -

$$
T=\frac{0,5(M)}{5000(\mathcal{M} / c)}=0,0001 \quad(c),
$$

шаг по времени (период волны) -

$$
\Delta t=\frac{T}{200}=5 \cdot 10^{-7} \quad(c) .
$$

При скорости движения возмущающей силы, равной 20 м/с, её время движения по рельсовой плети длиной 15 м составит: 


$$
t_{\text {дв }}=\frac{\ell_{\mathrm{p}}}{v}=\frac{15}{20}=0,75 c,
$$

где $l_{p}$ - длина рельса, $v$ - скорость движения возмущающего воздействия.

Пусть необходимо дополнительно рассмотреть свободные колебания на промежутке вре-

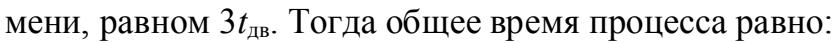

$$
T=4 \cdot t_{\text {дв }}=4 \cdot 0,75=3 \mathrm{c} .
$$

Учитывая, что шаг времени $\Delta t$ равен $5 \cdot 10^{-7} \mathrm{c}$, приходим к выводу, что необходимо сделать следующее количество выборок:

$$
n=\frac{t}{\Delta t}=\frac{3}{5 \cdot 10^{-7}}=6 \cdot 10^{6} .
$$

Кроме того, на каждом шаге необходимо просчитать параметры каждого элемента, число которых для стандартной рельсовой плети равно 27, следовательно, необходимо сделать

$$
27 \cdot n=27 \cdot 6 \cdot 10^{6}=1,62 \cdot 10^{8}
$$

количество одинаковых расчетов, что, очевидно, под силу лишь достаточно производительной ЭВМ.

Упростить задачу можно, применив наиболее эффективный формульный ряд с точки зрения арифметики ЭВМ. Это значит, что уравнения должны по возможности состоять из простых арифметических действий, не используя сложных математических функций, для которых ЭВМ использует отдельные встроенные алгоритмы.

Таким условиям, на наш взгляд, удовлетворяет система уравнений равновесия Д'Аламбера. Запишем ее применительно к нашей модели. Выделим элемент модели вместе с системой действующих на него сил (рис. 4).

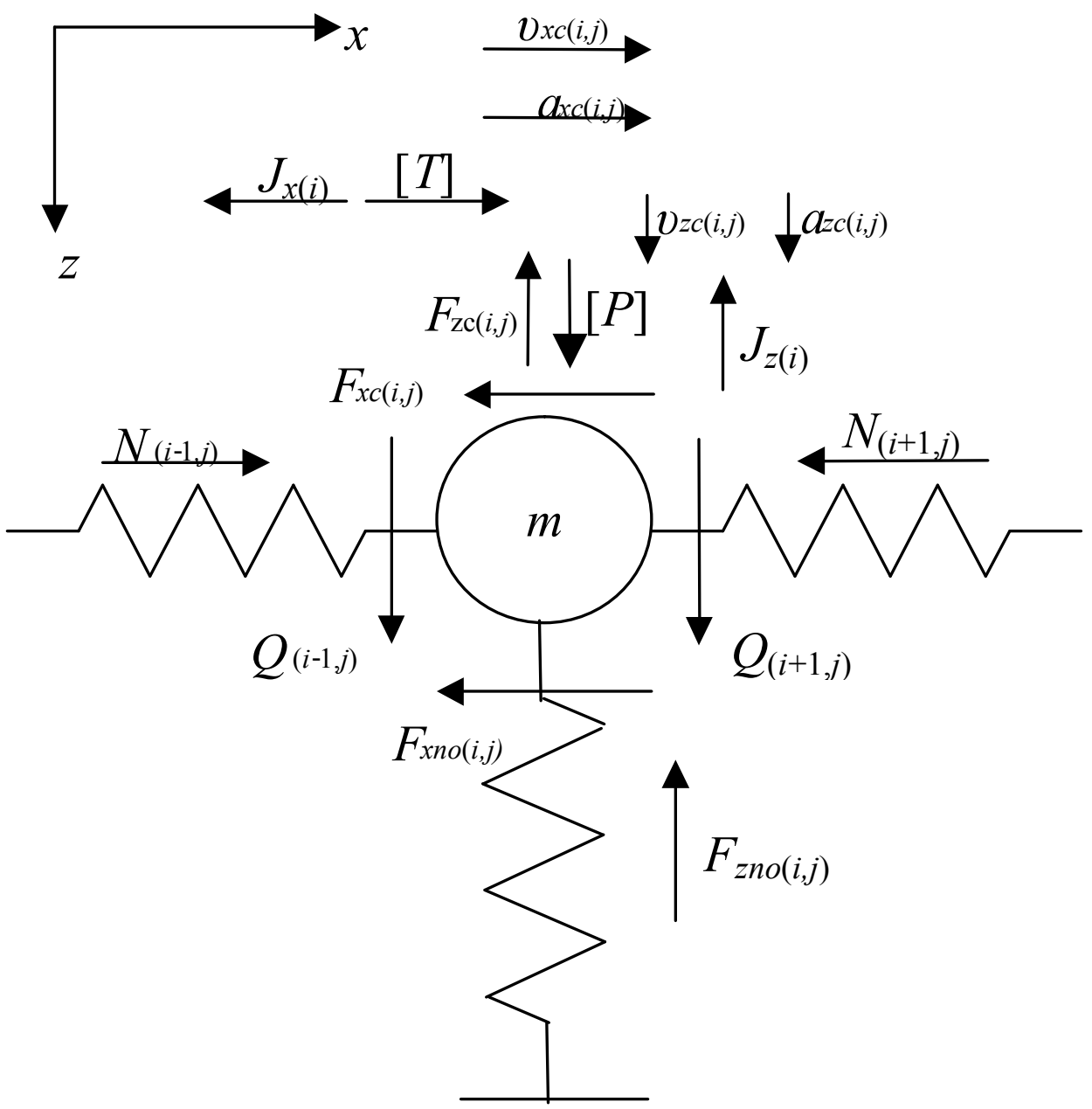

Р и с. 4. Силовая схема 
На рис. 4 введены обозначения: $[P]$ - внешняя вертикальная сила (вес состава); $[T]$ — горизонтальная внешняя сила, вызванная сопротивлением колес движению; $Q_{(i)}-$ поперечная (срезывающая) сила, возникающая из-за разницы в $z-$ координатах $(i-1)$ и $i$-того элементов; $Q_{(i+1)}$ - аналогично, для $i$ и $(i+1)$ - го элементов; $N_{(i-1)}$ - продольная сила вызванная деформацией рельса между $(i+1)$ и $i$-тым элементами; $N_{(i+1)}$ - то-же, для $i$-тых и $(i+1)$-тых элементов; $F_{x c(i)}$ и $F_{\mathrm{zc}(i)}$ - диссипативные силы, всегда направление противоположно вектору скорости; $F_{x n o(i)}$ и $F_{\text {zпо }(i)}$ - силы упругости подрельсового основания; $J_{x(i)}$ и $\mathrm{J}_{z(i)}-$ силы инерции.

Для каждого элемента может быть составлена система двух уравнений с двумя неизвестными $a_{x}$ и $a_{z}$ :

$$
\begin{aligned}
& -m a_{x(i, j)}+[T]_{(j)}-F_{x c(i, j)}-F_{x n n(i, j)}+N_{(i-1, j)}-N_{(i+1, j)}=0 ; \\
& -m a_{z(i, j)}+[P]_{(j)}-F_{z c(i, j)}-F_{z n n(i, j)}+Q_{(i-1, j)}+Q_{(i+1, j)}=0,
\end{aligned}
$$

откуда получим ускорения в виде

$$
\begin{aligned}
& a_{x(i, j)}=\frac{[T]_{(j)}-F_{x c(i, j)}-F_{x n n(i, j)}+N_{(i-1, j)}-N_{(i+1, j)}}{m} ; \\
& a_{z(i, j)}=\frac{[P]_{(j)}-F_{z c(i, j)}-F_{z n n(i, j)}+Q_{(i-1, j)}+Q_{(i+1, j)}}{m} .
\end{aligned}
$$

Исходя из поставленных ранее условий, свидетельствующих тому, что сила (возмущение) не могут передаваться от элемента к элементу мгновенно, можем заключить, что на промежутке времени $\Delta t$ система сил будет кинетостатической.

В этом случае найденные ускорения $a_{x i}$ и $a_{z i}$ будут также постоянны и будут отличаться от предыдущих не более чем на 0,03 , что обеспечивает достаточную точность аппроксимации.

Используя формулу для равноускоренного движения

$$
v=v_{0}+a \cdot \Delta t,
$$

определим скорость в конце интервала времени $\Delta t$, исходя из следующих граничных условий: конечная скорость в конце предыдущего интервала будет начальной в начале данного интервала, т.е.

$$
\begin{aligned}
& v_{x(i, j)}=v_{x 0(i, j)}+a_{x(i, j)} \cdot \Delta t ; \\
& v_{z(i, j)}=v_{z 0(i, j)}+a_{z(i, j)} \cdot \Delta t .
\end{aligned}
$$

Аналогичные граничные условия используем и для перемещений:

$$
\left\{\begin{array}{l}
\text { если а }=0, \text { то } \mathrm{S}=\mathrm{S}_{0}+v_{0} \Delta t ; \\
\text { иначе } S=S_{0}+\frac{v^{2}-v_{0}^{2}}{2 a} .
\end{array}\right.
$$

Применительно к нашей системе запишем:

$$
\begin{aligned}
& S_{x(i, j)}=S_{x o(i, j)}+\frac{v_{x(i, j)}^{2}-v_{x o(i, j)}^{2}}{2 a_{x(i, j)}} ; \\
& S_{z(i, j)}=S_{z o(i, j)}+\frac{v_{z(i, j)}^{2}-v_{z o(i, j)}^{2}}{2 a_{z(i, j)}} .
\end{aligned}
$$

Таким образом, на данном шаге были получены все интересующие нас параметры элемента модели, как-то: ускорения $a_{x}$ и $a_{z}$, скорости $v_{x}$ и $v_{z}$, перемещения $S_{x}$ и $S_{z}$ для каждого элемента системы. При переходе к следующему интервалу времени необходимо передать начальные условия. Это делается следующим образом:

$$
v_{x o(i, j+1)}=v_{z(i, j)}, v_{z o(i, j+1)}=v_{z(i, j)}, S_{x o(i, j+1)}=S_{x(i, j)}, S_{z o(i, j+1)}=S_{z(i, j)} .
$$

Силы, входящие в систему уравнений (7), определяются из условий

$$
\begin{gathered}
\bar{F}_{x c i}=-\kappa_{v} \cdot \bar{v}_{x o i}, \bar{F}_{z c i}=-\kappa_{v} \cdot \bar{v}_{z o i}, F_{x n o i}=S_{x o i} \cdot \aleph_{n o \leftrightarrow}, F_{z \text { поі }}=S_{z o i} \cdot \aleph_{n o \downarrow} ; \\
N_{i-1}=ж_{p \leftrightarrow} \cdot\left(\left[x_{i}-x_{(i-1)}\right]-\sqrt{\left[S_{x o i}-S_{x o(i-1)}+x_{i}-x_{(i-1)}\right]^{2}+\left[S_{z o i}-S_{z o(i-1)}\right]^{2}}\right) ; \\
N_{i+1}=\aleph_{p \leftrightarrow} \cdot\left(\left[x_{(i+1)}-x_{i}\right]-\sqrt{\left[x_{(i+1)}-x_{i}+S_{x o(i+1)}-S_{x o i}\right]^{2}+\left[S_{z o(i+1)}-S_{z o i}\right]^{2}}\right) ; \\
Q_{i-1}=-\varkappa_{p \downarrow} \cdot\left(S_{z o i}-S_{z o(i-1)}\right), Q_{i+1}=-\varkappa_{p \downarrow} \cdot\left(S_{z o i}-S_{z o(i+1)}\right), J_{x i}=-m a_{x 0 i}, J_{z i}=-m a_{z 0 i},
\end{gathered}
$$

где $\kappa_{v}$ - коэффициент пропорциональности, больший нуля; $\varkappa_{p}, \varkappa_{n o}-$ коэффициенты жесткости рельса и подрельсового основания соответственно. 
Если координата $x_{p}$ силы $P$ находиться между $x_{i}$ и $x_{(i-1)}$, то величина воздействия от силы $P$ на $i$ - тый элемент определяется как

$$
[P]=\frac{x_{p}-x_{i-1}}{x_{i}-x_{i-1}} P .
$$

Если координата $x_{p}$ силы $P$ находиться между $x_{i}$ и $x_{(i+1)}$, то величина воздействия от силы $P$ на $i$ - тый элемент определяется как

$$
[P]=\frac{x_{i+1}-x_{p}}{x_{i+1}-x_{i}} P .
$$

Если сила $P$ находится в окрестности $i$ - того элемента $\left(x_{p} \approx x_{i}\right)$, то $[P]=P$.

Сила продольного сопротивления движению равна:

$$
T=\omega_{0} P,
$$

где $\omega_{0}=2,5 \mathrm{H} /$ кН - основное удельное сопротивление движению.

На основе приведённого алгоритма была составлена программа для ЭВМ и произведены расчёты, анализ которых подтверждает справедливость предложенного метода моделирования. Полученные результаты во многом схожи с результатами аналогичных работ [2]. Наблюдались ярко выраженные колебательные процессы двух основных тонов: высокочастотные - с частотой собственных колебаний отдельных элементов, представляющих собой, в общем, физический пружинный маятник, и низкочастотные - колебания всей рельсовой плети в целом как единой системы.

\begin{tabular}{|c|c|c|c|}
\hline & Вариант 1 & Вариант 2 & Вариант 3 \\
\hline $\begin{array}{ll}\text { Вертикальная } & \text { жесткость } \\
\text { подрельсового } & \text { основания } \\
\varkappa_{n o} \uparrow, \mathrm{H} / \mathrm{M} & \\
\end{array}$ & $2,5 \cdot 10^{7}$ & $2,5 \cdot 10^{7}$ & $2,5 \cdot 10^{7}$ \\
\hline 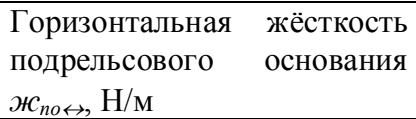 & $2 \cdot 10^{7}$ & $1,5 \cdot 10^{7}$ & $10^{7}$ \\
\hline $\begin{array}{l}\text { Максимальная сила удер- } \\
\text { жания рельсового скрепле- } \\
\text { ния } F_{\text {мах }}, \mathrm{H}\end{array}$ & 900 & 2000 & 2000 \\
\hline
\end{tabular}

Данные расчетов приведены в таблице, а графики на основании этих данных - на рис. 5-9.

Исходные данные

С целью упрощения вычислений (с сохранением наглядности работы алгоритма), все варианты расчетов приведены для десятишпального отрезка рельсовой плети, закрепленной в стыках.

На рис. 5-9 цифрами обозначено: 1 - график движения участка рельса над первой шпалой; 2 - график движения участка рельса над пятой шпалой; 3 - график движения участка рельса над десятой шпалой.

На графиках наглядно показано поведение различных участков рельса при прохождении по нему единичной силы, эквивалентной воздействию одной колёсной пары (для вагона с одноосной тележкой) или одной тележки вагона (с двухосной тележкой).

Анализ графиков указывает на то, что изменение горизонтальной жесткости подрельсового основания оказывает сильное влияние на характер протекающего колебательного процесса в горизонтальной плоскости и почти не оказывает влияния на характер вертикальных колебаний. С увеличением горизонтальной жесткости колебательный процесс приобретает более выраженные экстремумы, что говорит о больших внутренних силах, развиваемых в рельсе, и наоборот, снижение горизонтальной жесткости подрельсового основания делает график колебательного процесса более плавным.

Снижение прочности рельсового скрепления влечет за собой более раннее наступление колебательного процесса с проскальзыванием подошвы рельса по основанию. На графиках (рис. 8 и рис. 9), отражающих процессы при проскальзывании рельса отчетливо видны моменты наступления процесса угона. Данные моменты обуславливают появление «площадок» на графике колебательного процесса, что объясняется превышением внутренних сил в рельсе над силами удержания рельсового скрепления, когда происходит срыв подошвы рельса. При начавшемся 
процессе скольжения подошвы рельса по подрельсовому основанию силы трения скольжения уравниваются с вызвавшей этот процесс возмущающей силой («площадка»). Кроме того, повышается частота колебаний, равная при обычном колебательном процессе $40 \div 50$ Гц, при проскальзывании повышающейся до $450 \div 500$ Гц.

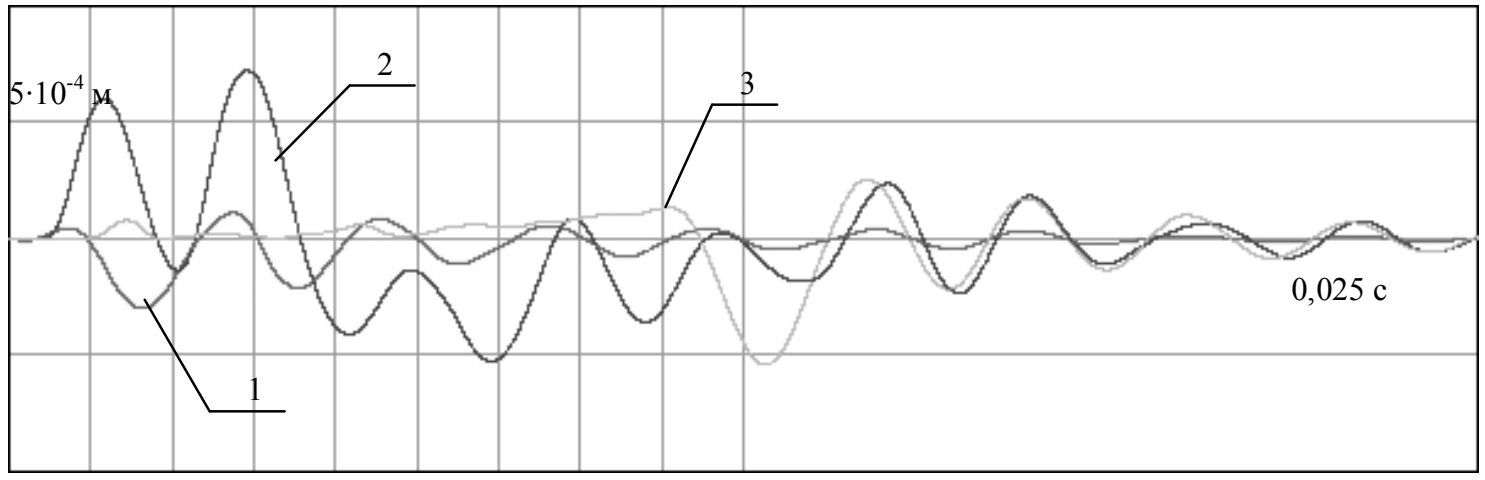

Р и с. 5. Перемещения подошвы рельса по горизонтали для первого варианта расчета (одно деление по вертикали $-5 \cdot 10^{-4} \mathrm{M}$, по горизонтали - $\left.0,025 \mathrm{c}\right)$ :

1 - перемещения для участка рельса над первой шпалой; 2 - перемещения для участка рельса над пятой шпалой; 3 - перемещения для участка рельса над десятой шпалой

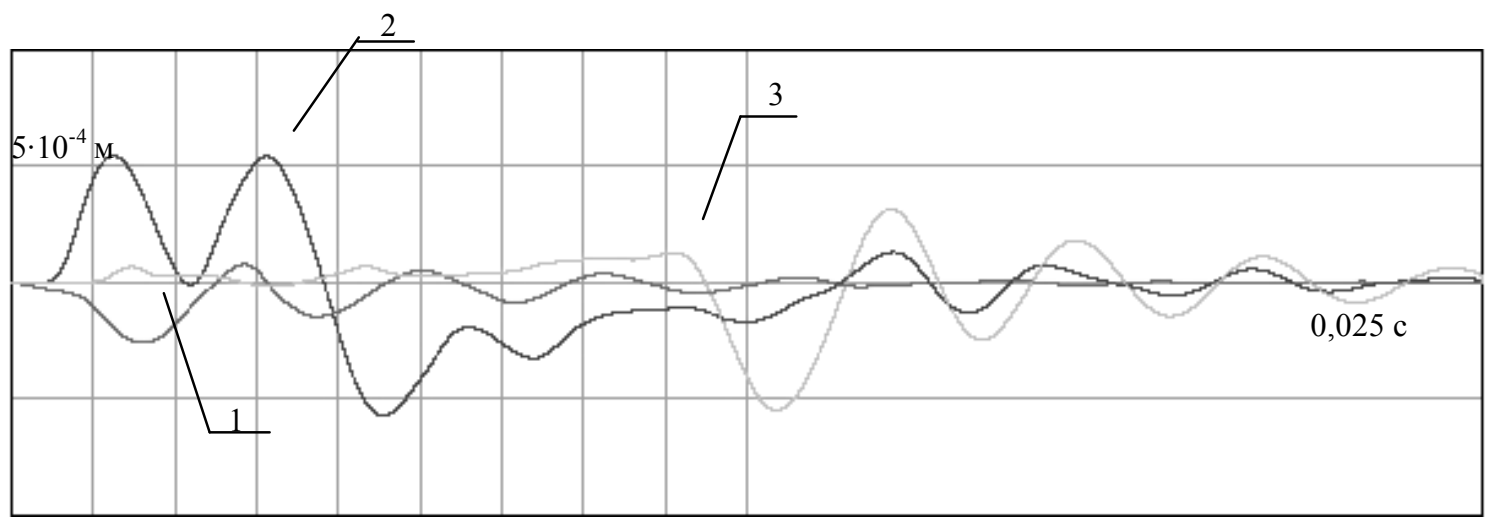

Р и с. 6. Перемещения подошвы рельса по горизонтали для второго варианта расчета (одно деление по вертикали $-5 \cdot 10^{-4} \mathrm{M}$, по горизонтали - $\left.0,025 \mathrm{c}\right)$ :

1 - перемещения для участка рельса над первой шпалой; 2 - перемещения для участка рельса над пятой шпалой; 3 - перемещения для участка рельса над десятой шпалой

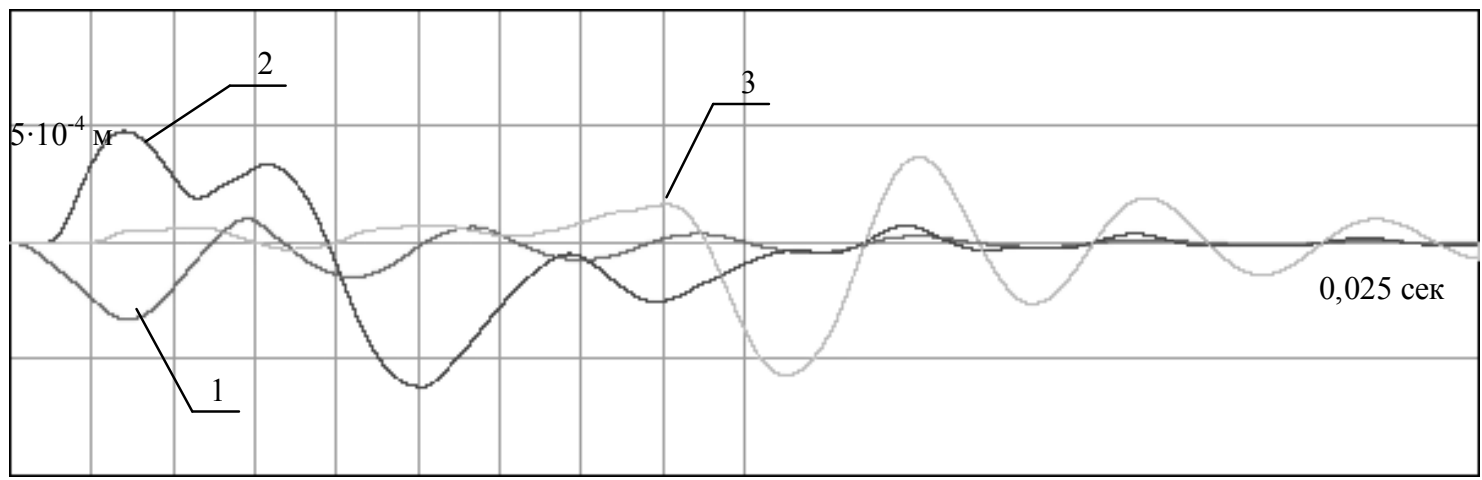

Р и с. 7. Перемещения подошвы рельса по горизонтали для третьего варианта расчета (одно деление по вертикали $-5 \cdot 10^{-4}$ м, по горизонтали $\left.-0,025 \mathrm{c}\right)$ :

1 - перемещения для участка рельса над первой шпалой; 2 - перемещения для участка рельса над пятой шпалой; 3 - перемещения для участка рельса над десятой шпалой 


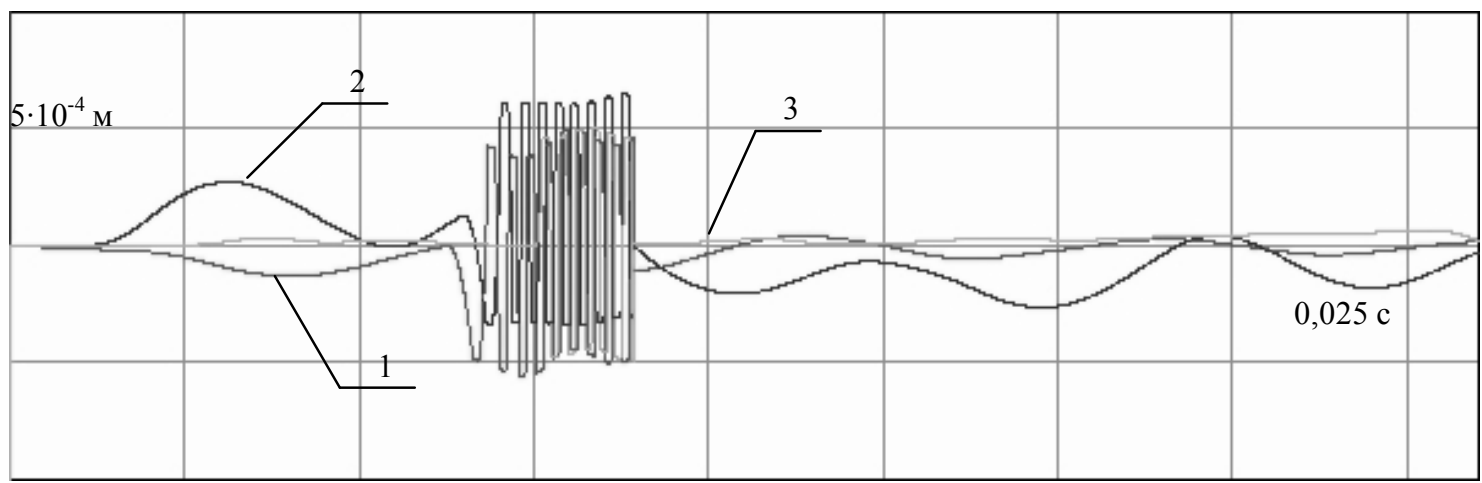

Р и с. 8. Перемещения верхней части подрельсового основания по горизонтали при проскальзывании рельса (одно деление по вертикали $-10^{-4}$ м, по горизонтали $-0,025 \mathrm{c}$ ):

1 - перемещения для участка рельса над первой шпалой; 2 - перемещения для участка рельса над пятой шпалой; 3 - перемещения для участка рельса над десятой шпалой

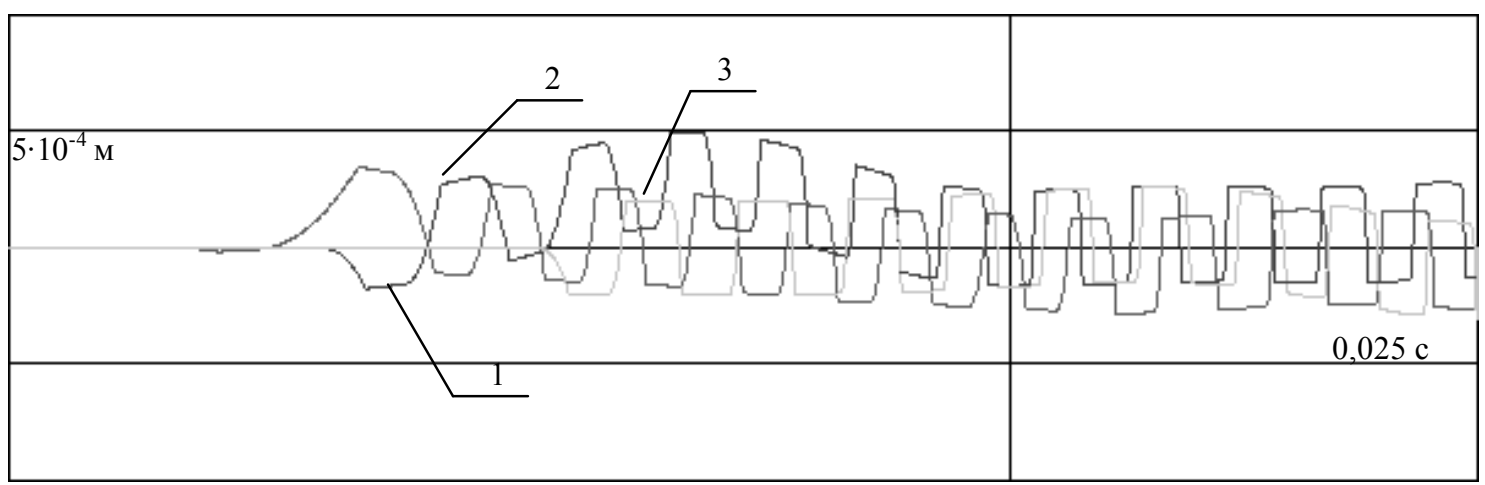

Р и с. 9. Искусственно смоделированные перемещения верхней части подрельсового основания по горизонтали при проскальзывании рельса при малой жесткости подрельсового основания и малой силе удержания в рельсовых скреплениях (одно деление по вертикали $-10^{-4}$ м, по горизонтали $-0,025$ с): 1 - перемещения для участка рельса над первой шпалой; 2 - перемещения для участка рельса над пятой шпалой; 3 - перемещения для участка рельса над десятой шпалой

Представленный выше упрощенный алгоритм расчёта и проведенные предварительные исследования причин угона и численной оценки его величины позволяют получить достоверные результаты и дать рекомендации по снижению силового воздействия, влияющего на угон пути.

\section{БИБЛИОГРАФИЧЕСКИЙ СПИСОК:}

1. Шахуньяни Г. М., Железнодорожный путь. М.: Транспорт, 1987. 479 с.

2. Меньшикова В. И. Динамические продольные силы и перемещения рельсов железнодорожного пути (угон рельсов) // Динамические исследования пути и корректировка правил расчётов железнодорожного пути на прочность. Труды ЦНИИ. Вып. 466. М.: Транспорт, 1972. С. 83-187. 